\title{
La prueba de la intención y la explicación de la acción
}

\author{
DANIEl GoNZÁlEZ LAGIER
}

Universidad de Alicante

\begin{abstract}
RESUMEN. En este trabajo se aborda el problema de la atribución de intenciones a otros sujetos, tratando de vincularlo, por un lado, con el problema filosófico de la explicación de las acciones humanas y, por otro lado, con el problema jurídico-procesal de la prueba del dolo. Se distingue entre dos tipos de concepciones, según asuman una postura cognoscitivista o no en relación con la validez de estas atribuciones, tomando postura a favor de la concepción cognoscitivista y proponiendo un esquema de razonamiento apropiado para la prueba judicial de la intención basado en un Principio de Racionalidad Mínima: esto es, se trata de mostrar la conexión entre intención y racionalidad y de sugerir que esta conexión puede usarse para probar la intención.
\end{abstract}

Palabras clave: Intención, prueba, explicación, racionalidad.

1. En este trabajo me ocupo de lo que llamaré la atribución de intenciones, esto es la determinación de la intención con la que un agente ha realizado una acción concreta. Así, un juicio por el cual se atribuye a un agente $A$ una intención es una respuesta a la pregunta "¿con qué propósito hizo $A$ la acción $x$ ?”.

2. El problema de la atribución de intenciones presenta múltiples facetas interesantes, algunas desde un punto de vista
AbStRACT. This article approaches the problem of attributing intentions to others, trying to relate that issue to, on the one hand, the philosophical problem of the explanation of human actions and, on the other, the procedural-law problem of the proof of criminal intent. Two kinds of conceptions are distinguished from each other, depending on whether they take up or not a cognitivist stance towards the validity of such attributions. The article supports the cognitivist conception, and proposes an outline for reasoning, appropriate for judicial proof of intention, that is based on a Minimal Rationality Principle: the point is to show the connection between intention and rationality, and to suggest that this connection can be used to prove intention.

Key words: intention, proof, explanation, rationality.

filosófico y otras desde un punto de vista jurídico (el clásico problema filosófico del conocimiento de las otras mentes, la determinación de la responsabilidad, la teoría de la acción y de la individualización de acciones, etc.). En este trabajo la atribución de intenciones me interesa en la medida en que es relevante, desde un punto de vista jurídico, para la prueba judicial de algunos tipos de hechos -las acciones intencionales y el dolo- $\mathrm{y}$, desde un punto de 
vista filosófico, para la explicación de la acción humana.

3. Comencemos con los problemas de prueba. Atribuir una intención a la acción de un agente es necesario en el marco de la prueba judicial de los hechos para -al menos- dos tipos de propósitos: Por un lado, para determinar -junto con la consecuencia de la acción- qué tipo de acción es la que el agente ha realizado (por ejemplo, establecer si al disparar se tenía la intención de matar o meramente de lesionar es una circunstancia relevante para la descripción de su conducta y su clasificación como un tipo u otro de acción). Por otro lado, para concluir si la acción fue realizada con dolo (y en qué grado) o imprudentemente, estableciendo de esta manera el grado de reproche que merece. Por tanto, los juicios de atribución de intenciones no sólo forman parte de la prueba del dolo, sino también de la prueba de que el agente realizó un tipo de acción determinado.

Ahora bien, las intenciones, como el resto de estados mentales o hechos internos (las emociones, las creencias, las sensaciones, etc.), tienen un modo subjetivo de existencia (no independiente del sujeto que los experimenta) y no son directamente observables por terceros; sólo el propio sujeto que los siente parece tener un conocimiento directo de los mismos (que ni siquiera puede ser considerado infalible), a través de lo que se ha llamado consciencia (por el contrario, los hechos externos tienen un modo objetivo de existencia y pueden ser observados por terceros). Estas peculiaridades -entre otras- han suscitado entre los filósofos muchas dudas acerca de si los estados mentales son hechos en el mismo sentido que los hechos externos, acerca de cómo "encajan" en la concepción científica del mundo y acerca de cómo pueden ser conocidos por terceros.
Ante estas dificultades, la doctrina procesal y la jurisprudencia sostienen que los hechos psicológicos no son susceptibles de prueba directa, sino de prueba indirecta o de indicios ${ }^{1}$. Esto es, nadie puede haber visto que un sujeto tenía una determinada intención (o una creencia, o una emoción), por lo que los estados mentales deben ser inferidos (o presumidos) a partir de la conducta externa del agente al que se atribuyen y de las circunstancias del contexto:

"La prueba de los elementos subjetivos del delito no requiere necesariamente basarse en las declaraciones testificales o en pruebas periciales. En realidad, en la medida en que el dolo o los restantes elementos del tipo penal no pueden ser percibidos directamente por los sentidos, ni requiere para su comprobación conocimientos científicos o técnicos especiales, se trata de elementos que se sustraen a las pruebas testificales y periciales en sentido estricto. Por lo tanto, el Tribunal de los hechos debe establecerlos a partir de la forma exterior del comportamiento $\mathrm{y}$ sus circunstancias mediante un procedimiento inductivo, que, por lo tanto, se basa en los principios de la experiencia general"'2.

Con este fin, la jurisprudencia ha ido creando un catálogo de "indicios-tipo" aptos para inferir el dolo en distintos tipos penales (por ejemplo, para la receptación se considera indicio de la intención que el precio de adquisición sea notablemente inferior al valor real de la cosa, y para la distinción entre "animus necandi" y "animus laedendi" la idoneidad del arma usada o la importancia vital del lugar del cuerpo al que se dirigió el ataque $)^{3}$.

Como puede imaginarse, este método indirecto de prueba de la intención, de determinación indirecta de la verdad 
de los enunciados acerca de los estados mentales, aunque parece inevitable, suscita enormes dudas y dificultades. ¿Podemos alcanzar de esta manera suficiente certidumbre acerca de las intenciones de los acusados o demandados para tomar estas decisiones? Las dudas son tantas que el Tribunal Supremo ha llegado a negar que los hechos psicológicos sean realmente hechos y los ha considerado "juicios de valor" de naturaleza subjetiva ${ }^{4}$ (lo que ha propiciado que sean tratados como parte de la quaestio iuris en lugar de materia de prueba); y parte de la doctrina penal -en una dirección semejante- ha señalado que la prueba del dolo no es en realidad una actividad cognoscitiva, sino una atribución o imputación a partir de los "indicios-tipo" 5 .

4. De esta manera, a propósito de la prueba del dolo o de la intención, se pueden identificar dos tipos de posturas o concepciones: Por un lado, las concepciones que podemos llamar cognoscitivistas o descriptivistas, para las que la prueba de la intención es descubrimiento, esto es, una actividad cognoscitiva, orientada a establecer juicios de atribución que son verdaderos o falsos. Para estas concepciones las intenciones son un tipo de realidad que es posible conocer, por lo que los criterios que debemos usar para formular nuestros juicios de atribución deben asegurarnos en la mayor medida posible la correspondencia entre nuestra atribución y esa realidad. Por otro lado, las concepciones adscriptivistas o normativistas, para las cuales la prueba de la intención es una imputación, esto es, una actividad que no tiende a descubrir ninguna realidad interna o psicológica (porque no existen los estados mentales, o porque no pueden ser conocidos, o porque no es necesario conocerlos en el proceso), sino a calificar de cierta manera la acción del agente de acuerdo con ciertos criterios que pueden satisfacer intereses muy variados (como el carácter ejemplificativo de la pena, la resolución de conflictos, etc.), pero no orientados al descubrimiento de la verdad.

5. Examinemos ahora cómo se plantea el problema de la atribución de intenciones en el ámbito de la discusión acerca del modelo metodológico adecuado para la explicación de la acción.

El punto de partida es tratar de responder a la siguiente pregunta: ¿Cómo deben explicarse las acciones humanas? ¿Cómo podemos explicar el hecho de que César cruzara el Rubicón, que un soberano declare una guerra, la aprobación de una ley en un parlamento o que mi amigo Pedro volviera la cabeza hacia otro lado cuando pasé cerca de él? Esta es una cuestión fundamental para las ciencias humanas, como la historia o la sociología, y está en el centro de la discusión acerca de si las ciencias humanas y las ciencias de la naturaleza comparten o no el mismo modelo de explicación científica (esto es, la discusión entre lo que podemos llamar monismo metodológico y dualismo metodológico, o positivismo y antipositivismo metodológico).

Explicar un acontecimiento o un hecho cualquiera $p$ es un acto de habla cuyo propósito es responder a la pregunta "¿por qué tuvo lugar $p$ ?"6. Una explicación de este tipo tiene, por tanto, la siguiente forma: " $p$ ha ocurrido porque $q " . q$ no puede ser cualquier cosa, sino que ha de guardar con $p$ cierta relación especial: una relación explicativa. Por supuesto, habría que indicar en qué consiste esta relación, pero eso depende de la teoría de la explicación que se asuma (y del tipo de explicación del que se hable). En todo caso, intuitivamente sabemos que hay ciertas cosas que resultan adecuadas para responder a la cuestión "por qué ha 
ocurrido $p$ " (todas ellas, como dice Searle, han de dar cuenta de la idea de "hacer que algo suceda"7) y otras que no resultan respuestas ni siquiera prima facie satisfactorias.

Ahora bien, a la pregunta "¿por qué Pedro volvió la cabeza hacia otro lado?" podemos responder de dos maneras: indicando las causas, en sentido mecánico, de ese hecho (por ejemplo, diciendo: "tiene una lesión muscular que le produce cierto tipo de espasmos") o dando las razones por las que lo hizo (por ejemplo, diciendo: "porque se ha enterado de que te han ascendido y creía que él lo merecía más"). Ahora bien, muchos autores aducen que estas explicaciones explican cosas distintas: la primera explica meramente ciertos movimientos corporales, pero no una acción; la segunda explica realmente una acción ${ }^{8}$ (y esto sería así incluso aunque las explicaciones se refieran al mismo tramo de conducta: un mismo comportamiento probablemente tiene siempre una explicación en términos de actividad neurofisiológica y otra en términos de razones, pero ambas explicaciones apuntan a dimensiones distintas del comportamiento). Esto es consecuencia de asumir un concepto de acción intrínsecamente relacionado con la idea de actuar por una razón o actuar intencionalmente. A las ciencias humanas les interesa la acción en cuanto que portadora de un significado, y no meramente como una serie de sucesos físicos y mecánicos. Otra posibilidad es responder que sí es posible dar una explicación en términos de relaciones causales de la acción realizada por razones, porque las razones son precisamente causa (en el sentido genuino) de las acciones. Es decir, podemos explicar una acción indicando las razones por las que el agente la realizó, pero eso no hace que la explicación deje de ser causal, porque las razones son causa de la acción ${ }^{9}$. Esta explicación sería, por tanto, al mismo tiempo causal e intencional ${ }^{10}$.

No me interesa entrar en la cuestión de si la explicación por razones es una explicación causal o teleológica o de un tipo peculiar. Lo que me interesa destacar es la convergencia de opiniones respecto a que las acciones (a diferencia de los meros movimientos corporales) se deben explicar señalando la intención con la que se actuó o las razones que las impulsaron ${ }^{11}$. Por tanto, la atribución de intenciones es esencial para una explicación de la acción que no sea meramente mecanicista, sino que dé cuenta de su significado.

6. Filósofos como Hempel y Popper han propuesto un modelo de explicación intencional de la conducta que toma como premisa general o ley de cobertura un Principio de Racionalidad ${ }^{12}$. Por ejemplo, el modelo nomológico-deductivo de Hempel (según el cual cualquier acontecimiento queda explicado cuando es la conclusión de un argumento deductivo que tiene como premisa general una ley y como premisa menor el explanans), puede adaptarse al caso de las acciones intencionales de la siguiente forma ${ }^{13}$ :

(1) $X$ deseaba $G$

(2) $X$ creía que hacer $A$, en ciertas circunstancias, es una (o la única, o la mejor) manera de lograr $G$

(3) Cuando un agente desea algo y cree que la realización de un cierto acto, en algunas circunstancias, es una (o la única, o la mejor) manera de satisfacer su deseo, realiza ese acto.

(4) Luego $X$ realizó $A$.

En este esquema, (3) enuncia el Principio de Racionalidad. Algunos autores consideran que el principio de Racionalidad tiene carácter empírico y, por tanto, falsable (Hempel); otros lo tratan como 
un presupuesto necesario para entender la conducta, pero que no informa acerca de cómo actúan de hecho las personas (Popper) ${ }^{14}$. A veces se considera un principio universal; otras veces, probabilístico. Pero tanto para unos como para otros la función del principio es la de permitir el paso de las intenciones y creencias a la acción efectivamente realizada (se trata de excluir la posibilidad de que el agente no actúe a pesar de tener las actitudes psicológicas adecuadas y de que no haya ningún impedimento). También es frecuente que los autores que proponen este modelo señalen la importancia de lo que Popper llamó la lógica de la situación. Con esta expresión se quiere destacar que la explicación debe hacerse teniendo en cuenta el marco físico y el entorno social e institucional en el que actúa el agente, y su evaluación de todas estas variables ${ }^{15}$.

Los modelos propuestos por Hempel

y Popper toman postura a favor del monismo metodológico en la discusión acerca de cuál es el método propio de las ciencias de la conducta. El Principio de Racionalidad opera como las leyes causales naturales; el tipo de explicación es en sustancia el mismo para las ciencias de la naturaleza y las ciencias del hombre. Von Wright ha propuesto, por el contrario, un modelo distinto (sin Ley de Cobertura, aparentemente) en su defensa del dualismo metodológico. Su propuesta es que el silogismo práctico aristotélico puede ofrecer el patrón de explicación propio de la acción humana. El siguiente sería el esquema del silogismo práctico:

(1) $A$ tiene intención de $p$

(2) $A$ juzga que a menos que haga $q$ no será capaz de dar lugar a $p$.

(3) Por consiguiente, $A$ se dispone a hacer $q$.

"Es fácil percatarse -dice von Wrightde lo que tiene que ver el razonamiento práctico con la explicación de la acción. Supongamos que $A$, de hecho, hace $q$. Deseamos saber por qué. Una respuesta satisfactoria a esta cuestión sería señalar que tenía la intención de $p$ y que consideraba hacer $q$ necesario para este fin. Y esta misma respuesta explicaría por qué trató de hacer $q$ en una situación en la que terminará fracasando"16 . En realidad, la propuesta de von Wright parece diferir de la de Hempel y Popper sólo en la (aparente) ausencia del Principio de Racionalidad. En su lugar, el modelo de von Wright asume que podemos inferir una acción a partir de creencias y deseos (intenciones) por razones conceptuales: "Decir que tales y cuales intenciones y creencias, en el caso de que no cambien, darán normalmente como resultado tal y cual conducta no es establecer una generalización empírica basada en observaciones y experimentos. Es establecer una verdad necesaria que aceptará sin dar más vueltas todo aquél que se halle familiarizado con los conceptos involucrados"17. De manera que la regla de inferencia que nos permite inferir la acción a partir de las creencias y los deseos tiene carácter conceptual ${ }^{18}$.

7. Sin embargo, ni uno ni otro modelo de explicación ${ }^{19}$ dan cuenta de todas las fases de la explicación intencional de una acción. No se refieren a cómo obtenemos el deseo y las creencias que usamos para explicar la acción (salvo la indicación sobre la importancia de tener en cuenta la lógica de la situación), sino sólo de cómo queda explicada la acción una vez que tenemos esos datos ${ }^{20}$. En otras palabras: nos dicen que una acción queda correctamente explicada desde un punto de vista intencional cuando puede inferirse de una premisa sobre las actitudes volitivas y epistémicas del agente y una premisa general (de carácter empírico, conceptual...) que viene a establecer 
que los agentes actúan de acuerdo con sus deseos y creencias. Ahora bien, para que la explicación sea correcta, la premisa que establece cuáles son los deseos y creencias del agente debe ser verdade$\mathrm{ra}^{21}$. ¿Cómo podemos saber que lo es? Al preocuparnos por la justificación externa de la explicación, el problema de cómo conocer los estados mentales internos de los agentes aparece de nuevo, y se muestra que los modelos de explicación intencional de la conducta no lo afrontan (un modelo completo de explicación de la conducta debería, sin embargo, incluirlo). En definitiva: a estos modelos parece faltarles un análisis del proceso de atribución de intenciones.

8. Lo dicho hasta ahora no permite todavía tomar postura acerca de si la atribución de intenciones es una operación cognoscitiva o adscriptiva. En este trabajo sostendré que los juicios de atribución de intenciones son verdaderos o falsos, esto es, aceptaré la concepción cognoscitivista. En defensa de esta postura comentaré brevemente los argumentos contra el cognoscitivismo y propondré un modelo de atribución de intenciones, tratando de mostrar que este modelo se fundamenta en su éxito para descubrir las intenciones de los demás.

9. Si examinamos los argumentos contra la concepción cognoscitivista podemos clasificarlos en tres tipos: ontológicos, epistemológicos e ideológicos.

Los argumentos de tipo ontológico, en su rechazo del dualismo cartesiano, proponen la eliminación de toda referencia a lo mental o al menos la eliminación de algunas de sus propiedades más características, como la consciencia. Se trata de posturas como el conductismo lógico (que sustituye los estados mentales por disposiciones de conducta externa), el materialismo eliminacionista (que lo sustituye por estados cerebrales físicos) o la tesis de la "postura intencional" de Dennet (que postula que los estados mentales son sólo el resultado de adoptar cierta perspectiva para explicar las acciones, pero no son fenómenos intrínsecamente mentales). La principal crítica que puede hacerse a estas tesis es que nos piden aceptar conclusiones muy contraintuitivas, cuando en realidad su fundamentación todavía es una cuestión abierta. Podemos suponer una tesis acerca de la justificación de las teorías que prescribe asignar cierto peso a nuestras intuiciones. Las intuiciones y creencias de sentido común no tienen por qué ser un límite infranqueable para nuestras teorías -ni siquiera las intuiciones básicas-, pero tampoco pueden ser sencillamente ignoradas (especialmente las más básicas). Como diría Rescher, merecen respeto, aunque no necesariamente aceptación ${ }^{22}$. Sin embargo, estas teorías rechazan las intuiciones sobre lo mental con el argumento general de que no cuadran con ellas mismas, sin dar argumentos independientes para su rechazo. El argumento que se repite una y otra vez es que no caben en la teoría, pero eso -a falta de certeza acerca de la teoría- no es suficiente ${ }^{23}$.

Los argumentos epistemológicos señalan que la prueba de la intención es poco fiable o imposible. Hay distintas razones para afirmar esto: los juristas suelen decir que la prueba indirecta o de indicios, que es la única posible para probar los estados mentales, es menos fiable que la prueba directa; otras veces se sostiene que no es posible encontrar regularidades que conecten la conducta externa con las intenciones, porque una misma intención puede ser satisfecha por medio de comportamientos distintos; otras veces se sostiene que no se dispone de un método de verificación de la corrección de la prueba de la intención 
más allá de la propia prueba (¡como si en la prueba judicial esto fuera posible en algún caso!) o que cuando atribuimos intenciones a los demás nos limitamos a proyectar las intenciones que nosotros hubiéramos tenido en esa situación. Todos estos argumentos parecen suponer que puesto que no es posible demostrar sin margen de error qué intención tuvo un agente, debe desistirse de intentarlo. Ahora bien, si nos tomáramos en serio estos argumentos y los generalizáramos coherentemente nos llevarían también a desistir de casi todo intento de conocer cualquier cosa. Este tipo de argumentos incurren por lo general en la misma falacia: pasan de la afirmación (correcta) de que no es posible tener una certeza absoluta acerca de la corrección de la atribución de una intención a un agente a la conclusión (equivocada) de que no tiene sentido perseguir la verdad en este ámbito (algo semejante hacen quienes niegan que la verdad deba buscarse en el proceso, puesto que la verdad absoluta es inalcanzable).

El tercer grupo de argumentos, los argumentos ideológicos, aduce que los fines del proceso hacen innecesario o, incluso, inconveniente, tratar de descubrir las intenciones reales de los agentes. Esto es, no sostienen que la intención no pueda ser descubierta, sino que no debe o no tiene por qué ser descubierta. Así, por ejemplo, las concepciones persuasivas de la prueba niegan que ésta sea un instrumento de conocimiento de la verdad (o de la verdad como correspondencia con la realidad) y la presentan como un instrumento de persuasión o convencimiento del juez (o para llegar a algún otro tipo de "verdad", como la "verdad formal"). Estas tesis van unidas a una concepción del proceso como medio de resolución de conflictos, en donde no importa tanto la justicia de la decisión como alcanzar una solución. Este argumento general puede ser refutado, como hace Taruffo, mostrando, por un lado, que la concepción persuasiva de la prueba es sólo una visión parcial del funcionamiento y los objetivos del proceso (pues pone el acento en la perspectiva del abogado, olvidando la perspectiva del juez) y señalando, por otro lado, la necesidad, para que la decisión judicial sea justa, de que se hayan comprobado, en la mayor medida posible dentro del contexto judicial, que efectivamente se han producido los hechos que se describen en el supuesto de hecho de la norma (lo que requiere una teoría de la verdad como correspondencia en el ámbito de la prueba jurídica). Otras veces se sostiene -como lo hace Jakobs- que la finalidad esencial de la pena es asegurar a la sociedad que la norma violada sigue vigente, por lo que no puede permitirse el caso de que la sociedad considere que una norma ha sido violada intencionalmente y no se siga la pena pertinente, con independencia de que hubiera intención real o no de violar la norma, de donde se infiere que lo importante no es la intención real del agente, sino cómo va a considerar (intencional, o no) la sociedad su conducta. Una sentencia en la que se declare que no se actuó intencionalmente no será comprendida si desde el punto de vista social todo apunta a que se había actuado intencionalmente. En palabras de Laurenzo Copello (refiriéndose específicamente a Puppe, pero con referencias también a Jakobs): "Lo importante, por consiguiente, de cara a determinar si se produce una contradicción con la norma jurídico penal, no son las actitudes internas del sujeto, sino lo que exprese la conducta desde la perspectiva de la comunicación entre seres inteligentes". Sin embargo, este tipo de argumentos es susceptible de una objeción importante: dependen de que no se le dé ningún valor al reproche como justificación de la 
pena, porque si éste tuviera algún valor, estaríamos asumiendo implícitamente que la intención que nos interesa es la real (como estado mental que causó la acción del sujeto). Y aunque puede quizá aceptarse que la explicación de la pena es la afirmación de la vigencia de la norma, la prevención general, positiva o negativa, o algún tipo de finalidad de este estilo, es difícil negar que la justificación de la misma tiene sus raíces en las ideas de libre albedrío y reproche, que constituyen un presupuesto del sistema penal, y así se percibe incluso por la conciencia social a la que estas teorías aluden.

Por otro lado, si enfocamos estas objeciones al cognoscitivismo desde el punto de vista de la explicación de la acción, nos encontramos con que negar la posibilidad de conocer las intenciones reales de los sujetos parece implicar la negación de la posibilidad de dar explicaciones satisfactorias de la misma: Sólo podemos explicar una acción si señalamos la razón por la que realmente el agente hizo lo que hizo (su intención real). No basta con señalar que había una razón para actuar, sino que hay que postular que el agente actuó movido por esa razón. Quienes niegan la posibilidad de describir con cierto grado de objetividad las intenciones (u otros estados mentales relevantes), deben rechazar la posibilidad de explicar las acciones a la luz de razones (más adelante trataré de mostrar que actuar intencionalmente es actuar por una razón). Deberán explicar la conducta en términos causales cuyo antecedente no sean intenciones, razones, deseos, etc., sino estados neuronales o compulsiones físicas. No explicarán, en definitiva, acciones, sino movimientos corporales.

10. Resta construir un modelo de atribución de intenciones. Para ello creo que el camino es el de profundizar en el sentido del Principio de Racionalidad. En primer lugar, trataré de precisar a qué acciones es aplicable; en segundo lugar, trataré de encontrar el mínimo de racionalidad que necesariamente contienen las acciones intencionales y de formular el Principio de Racionalidad con mayor precisión; en tercer lugar, mostraré cómo se inserta este principio en el argumento que concluye en la atribución de una intención. De lo que se trata, en definitiva, es de determinar si todas las acciones intencionales son racionales en un sentido mínimo; si fuera así, la adecuación racional de una acción respecto de un fin se convertiría en el criterio más relevante para atribuir una intención a esa acción.

11. No todas nuestras acciones son intencionales, y no todas las acciones que consideramos intencionales lo son en el mismo sentido:

a) Un primer grupo de acciones, los actos reflejos, son aquellos que se producen sin intervención de la voluntad. Son reacciones que no se encuentran dirigidas por la intención o el propósito de alcanzar un determinado resultado. Además, no podemos controlarlas: quizá las podemos realizar intencionalmente, pero no reprimirlas.

b) A un segundo grupo de acciones, lo podríamos llamar "acciones expresivas". Son expresiones de alguna emoción o algún estado de ánimo, que las causa. Por ejemplo, es posible que si estoy sentado esperando nervioso algún acontecimiento importante en mi vida, de repente me levante y me ponga a pasear y silbar por la habitación, sin haberlo decidido. $\mathrm{O}$ si en medio de una discusión acabo enfadándome es posible que empiece a manotear. Se trata de acciones que expresan algún estado 
de ánimo, pero sin que yo me haya propuesto expresarlo. ¿Son acciones intencionales? Yo podría hacerlas intencionalmente, y podría aprender a evitarlas; es decir, en mayor o menor medida, puedo controlarlas. $\mathrm{Si}$ me doy cuenta de que me voy a levantar y ponerme a silbar, puedo evitarlo. Además, mientras las estoy haciendo, o cuando alguien me pregunta qué estoy haciendo, soy consciente de ellas (en el sentido de que tengo una certeza no basada en la observación de mi conducta externa de lo que estoy haciendo). Sin embargo, no puedo dar una razón (finalista, no meramente causal) de por qué las estoy haciendo. Todo esto nos autoriza a decir que son intencionales, pero en un sentido mínimo o débil. A veces expresamos lo mismo diciendo que son voluntarias, para distinguirlas de los meros actos reflejos.

c) Un tercer grupo está constituido por las acciones intencionales en sentido estricto o dirigidas a fines. Estas se dirigen a un objetivo y en ellas intervienen deseos y creencias acerca de cómo satisfacerlos. En algunos casos, el carácter intencional de estas acciones es más evidente, porque son el resultado de una decisión razonada: Deliberamos acerca de qué fines perseguir y cuáles son los medios adecuados para alcanzarlos. Otras veces realizamos una conducta dirigida a un fin sin que hayamos deliberado previamente y sin haber decidido, en ese momento, hacerla; aun así, también estas acciones son intencionales: queremos el fin y queremos la acción como un medio para conseguirlo. Cuando salgo de mi casa por las mañanas y conduzco hasta la universidad, realizo muchas acciones de este tipo (darle la vuelta a la llave de casa, andar, arrancar el coche, insertar la primera, poner el intermitente, mirar por el retrovisor, etc., etc., etc.). Aunque no sean el resultado de una decisión en cada momento previo a su realización, son el producto de un proceso de aprendizaje o de surgimiento de un hábito, y si retrocedemos en el tiempo a lo largo de ese proceso, acabamos encontrando en su origen un esfuerzo para realizarlas y, probablemente, una decisión.

d) Un cuarto grupo (o, si se quiere, un subgrupo del anterior) está constituido por aquellas acciones intencionales que no se dirigen a un fin distinto de la mera realización de la propia acción. Mosterín habla de acciones que tienen ellas mismas un sentido final o de fin: no las realizo para obtener un fin distinto, sino que ellas mismas son el fin que persigo. Por ejemplo, cantar por cantar o leer un libro por placer. Sin embargo, podemos decir (aunque resulte un poco artificioso) que también estas acciones persiguen un fin: satisfacer mi deseo. Deseo (porque me apetece) cantar y sé que la mejor manera de satisfacer mi deseo es ponerme a cantar. Las trataré, por tanto, como acciones dirigidas a fines (no obstante, es importante retener que no siempre que hacemos algo intencionalmente perseguimos un fin distinto a la mera realización de la acción intencional).

e) Y un quinto grupo estaría constituido por las acciones no intencionales. Éstas son siempre (salvo en el caso de los actos reflejos, y si dejamos fuera de este grupo a las acciones expresivas) consecuencias no queridas y no previstas de otra acción. Así, si atropello a alguien al saltarme un semáforo, el atropello no es intencional, aunque saltarme el semáforo sí lo haya sido; y si tiro el jarrón al suelo 
al intentar encender la luz, haber tirado el jarrón es una acción no intencional consecuencia de mi acción intencional de intentar encender la luz.

Las siguientes consideraciones son válidas sólo para las acciones del tercer grupo, a las que llamará intencionales en sentido estricto. En este sentido, actuar con una intención es actuar persiguiendo un fin. Podemos decir que procurar ese fin es la razón de nuestra acción. Por tanto, podemos asumir la siguiente definición: $X$ hace $A$ con la intención de dar lugar a $F$ si cree que $A$ es un medio para dar lugar a $F$ y hace $A$ por esa razón. Actuar intencionalmente también se puede definir como actuar por una razón.

12. También el término "racionalidad" es ambiguo, por lo que para determinar en qué sentido toda acción intencional es racional debemos deshacer primero esa ambigüedad. Podemos distinguir tres sentidos de "racionalidad":

a) Racionalidad perfecta: Un agente actúa de manera perfectamente racional cuando lo hace movido por razones objetivamente buenas. En el momento de actuar, lo que consideró buenas razones lo eran objetivamente (esto es, para cualquier ser racional).

b) Racionalidad imperfecta: Un agente actúa de manera imperfectamente racional cuando lo hace movido por las razones que a él le parecen buenas, si éstas no coinciden con las objetivamente buenas. Se trata de razones que le parecerían buenas a cualquier persona como él, pero no a toda persona racional. Por ejemplo, si deseo ir a Barcelona en tren en lugar de tomar el avión porque soy supersticioso y tengo que viajar en martes 13, mis razones serán buenas para mí (y los supersticiosos), pero no son objetivamente buenas. Sin embargo, dado que hay una coherencia entre mis creencias generales y mis acciones, podemos hablar de algún grado de racionalidad.

c) Racionalidad mínima: un agente actúa de manera mínimamente racional cuando lo que creyó en el momento de actuar que eran buenas razones no lo eran objetivamente, y además tampoco coincidían con lo que en condiciones normales él mismo hubiera considerado buenas razones. Se trata de supuestos de error en la ocasión concreta en que se actuó por falta de deliberación, urgencia de actuar, defectuosa interpretación de las circunstancias, uso de información no fiable, desprecio de información relevante, exceso de emotividad, etc. Pero incluso en estos casos hubo cierta coherencia entre mis creencias efectivas en el momento de actuar y mi acción: ésta se ajustó a las razones que creía adecuadas en ese momento, aunque fueran equivocadas.

Creo que el siguiente pasaje de John Watkins, aunque largo, puede contribuir a aclarar qué entiendo por racionalidad mínima (que él llama racionalidad imperfecta):

"Al conjunto de todas aquellas consideraciones que, se formulen o no conscientemente, entran en un caso particular de toma de decisión lo llamo 'esquema de decisión'. Según la teoría normativa de la decisión, un esquema de decisión debe consistir en una especificación completa de valores de retribución a los resultados posibles, un mapa de preferencias completo o una asignación completa de valores de retribución a los resultados, y (en los casos en que resulta adecuado) un sistema para hacer frente a los diversos riesgos e incertidumbres. 
Si se le juzga por esto, un esquema de decisión real es usualmente algo verdaderamente muy imperfecto. Un esquema de decisión ideal se describe como algo que la mente del agente tiene presente en su totalidad, un todo completo en el que los varios componentes juegan simultáneamente su papel oportuno. Un esquema de decisión real se construye generalmente parte a parte, de manera que la llegada de una parte aislada de información situacional puede tener una influencia totalmente desproporcionada. E incluso cuando estén incluidos todos los datos, la significación práctica de las diferentes partes del mismo puede crecer o disminuir a medida que el que ha de tomar la decisión atiende ora a un factor, ora al otro.

No solamente es un esquema de decisión real más o menos vago y fragmentario cuando se le compara con el ideal, sino que el agente lo reducirá y simplificará aún más a medida que se acerca una decisión. En lugar de la enumeración completa de las posibilidades que exige la teoría normativa, nos fijamos en unas cuantas características y elegimos algunas posibilidades interesantes de la situación-problema dada"

Es obvio que no toda acción intencional es perfectamente racional: en muchas ocasiones fallamos en alcanzar nuestros objetivos por una inadecuada elección de los medios. Tampoco podemos decir que las acciones intencionales sean siempre al menos imperfectamente racionales, porque puede haber errores en nuestros cálculos que nos hagan actuar de una manera contraria a como nosotros mismos hubiéramos actuado de haber pensado las cosas con más calma. ¿Podemos decir al menos que todas las acciones intencionales son mínimamente racionales? Para llegar a esto todavía tenemos que hacer una restricción más.
13. Como sabemos, la racionalidad puede ser de fines ( $₫$ son adecuados los fines y objetivos del agente?) o instrumental (¿son adecuados los medios escogidos para alcanzar los fines perseguidos?). Creo que debemos admitir que no siempre actuamos persiguiendo lo que creemos, en un momento concreto, que son los mejores fines, los más adecuados o los que más nos convienen. A veces -muchas veces- actuamos siendo conscientes de que nuestros fines no son los que debemos perseguir. "Demasiado a menudo -dice Rescher- los deseos y apetitos nos guían en lo que hacemos, y éstos pueden ser o no buenos consejeros con respecto a la racionalidad (...) Los individuos automáticamente tienen un motivo cuando se presenta un deseo, pero sólo tienen buenas razones cuando evidentemente hacen algo de acuerdo con su mejor interés". De manera que, aceptado esto y dejando de lado por tanto la racionalidad de fines, lo que nos queda de la pregunta anterior es lo siguiente: ¿siempre que actuamos intencionalmente soy al menos mínimamente racional desde el punto de vista instrumental, esto es, me muevo para satisfacer un deseo y elijo los medios que me parecen más adecuados en ese momento para satisfacerlo?

Creo que la respuesta es necesariamente afirmativa. Es una cuestión conceptual que cuando actúo con la intención de dar lugar al fin $F$ realizo la acción que en ese momento me parece más adecuada (teniendo en cuenta mis posibilidades, mis preferencias, mis meros "gustos" y mi evaluación de la situación) para lograr $F$. En eso consiste actuar intencionalmente. Obsérvese que no se trata de elegir una acción adecuada cualquiera, sino la que considero la más adecuada. Si elijo una acción que puede contribuir al resultado, pero no es la más adecuada, y no puedo aducir ninguna 
razón o preferencia para haberla escogido (en un sentido muy amplio, que puede incluir el mero hábito), ningún observador dirá que tuve la intención de producir $F$ con mi acción (salvo que se convenza de que hay alguna razón oculta para mi preferencia); y yo mismo no podré decirlo seriamente. De manera que la racionalidad que encontramos en todas las acciones intencionales es una racionalidad instrumental mínima, lo que implica que cuando hacemos una acción intencional consideramos que tenemos una buena razón (instrumental) para hacerla. Llamaré a esta conexión entre acción intencional y racionalidad Principio de Racionalidad Mínima (PRM) . El PRM puede enunciarse de la siguiente manera: "Si un agente actúa intencionalmente (y, por tanto, persigue un fin), siempre realiza la acción que cree más adecuada para lograr el fin que persigue". En el siguiente apartado trataré demostrar cómo se integra este principio en el razonamiento probatorio de las intenciones.

14. ¿Cómo podemos aplicar el PRM para la atribución de una intención? Veámoslo con un ejemplo: Alfredo y viven en huertas colindantes. Una tarde, Alfredo llama a grandes voces a Herminio, con el que anteriormente había mantenido múltiples y acaloradas discusiones. Al asomarse éste a la puerta de su casa entablan una fuerte discusión sobre la propiedad de una vereda que separa ambas fincas. La discusión es interrumpida por el yerno de Herminio, quien asiéndole del brazo lo mete en la casa. A continuación, Alfredo entra en su propia casa, coge una escopeta IS, modelo PR, del calibre 12 y vuelve a salir, retando a Herminio a gritos; le sigue su hermana Arsenia, quien, agarrándole del brazo, trata de disuadirle. Cuando Herminio se asoma de nuevo a la puerta, Alfredo le dispara un cartu- cho de perdigones desde una distancia de unos 12 metros. En el momento en que Alfredo dispara, Arsenia tira de él hacia atrás para desviar la trayectoria del disparo. Los perdigones impactan en un muro de piedra que está detrás de Herminio (a un metro de altura), sin que éste resulte lesionado. Se calcula que la trayectoria del disparo se ha desviado 70 $\mathrm{cm}$. respecto de la que hubiera matado o herido a Herminio. Estos hechos son calificados por el tribunal que los juzga como un delito de homicidio en grado de tentativa, lo que significa que se da por probado (a) que la acción de Alfredo es intencional y (b) que su intención es la de matar a Herminio y no meramente lesionarle (en cuyo caso se le podría haber condenado de un delito de lesiones en grado de tentativa) ${ }^{24}$.

¿Cómo sabemos que Alfredo disparó con la intención de matar a Herminio, y no meramente de lesionarle? La respuesta es que, de acuerdo con el Principio de Racionalidad Mínima, los sujetos procuran realizar la acción que, a su juicio, tiende a asegurarles el resultado que pretenden y la acción de Alfredo fue instrumentalmente adecuada para matar a Herminio (como sabemos por el calibre de la escopeta usada, el lugar del cuerpo de Herminio hacia donde apuntó, la distancia, etc.).

El razonamiento completo sería el siguiente:

(1) Los agentes realizan la acción que creen más adecuada para lograr el fin que persiguen (PRM).

(2) Alfredo creía que la manera más adecuada de matar a Herminio consistía en disparar en las circunstancias $\mathrm{C}$.

(3) Alfredo disparó en las circunstancias C.

(4) Alfredo disparó intencionalmente, esto es, con un fin ulterior (presunción de intencionalidad). 
(5) Alfredo disparó con el fin de matar a Herminio (esto es, tenía la intención de matar a Herminio).

En general, el argumento por el que se atribuyen intenciones puede reconstruirse de la siguiente manera:

(1) Los agentes realizan la acción que creen más adecuada para lograr el fin que persiguen (PRM).

(2) S creía que la manera más adecuada de conseguir $\mathrm{F}$ consistía en hacer A en las circunstancias $\mathrm{C}$.

(3) S hizo A en las circunstancias C.

(4) S hizo A intencionalmente (presunción de intencionalidad).

(5) S hizo A con la intención de conseguir $\mathrm{F}$.

Obsérvese que la premisa 4 establece una presunción de intencionalidad: cuando observamos los movimientos corporales de un sujeto, presumimos que son realizados con alguna intención, y a la luz de esa presunción buscamos el sentido de su acción. Sólo si no lo encontramos, empezaremos a preguntarnos si tal acción no fue intencional, esto es, si fue un acto reflejo o algo que hizo sin la guía de la voluntad; por el contrario, si encontramos un propósito que dé sentido a la acción y la haga aparecer como mínimamente racional, confirmamos la presunción de intencionalidad. Por tanto, presumimos que una acción no es intencional cuando no entendemos su sentido, a la luz del PRM.

15. ¿Es éste un razonamiento deductivo? ¿Pueden ser aceptadas las premisas y rechazada la conclusión? ¿Es lógicamente posible que Alfredo quisiera meramente lesionar a Herminio, aunque de hecho le apuntara al corazón? Esto sería posible en dos situaciones: si se ha disparado al corazón por accidente, o involuntariamente, $\mathrm{o}$ bien si se desconocía que disparar al corazón era un medio adecuado para matar. Pero lo primero queda descartado en nuestro argumento por la premisa (4) y lo segundo por la premisa (2). De manera que el argumento es concluyente. Es lógicamente imposible disparar al corazón intencionalmente (no por accidente o error, sino con un propósito ulterior), creer que hacer tal cosa es el medio más adecuado en esas circunstancias para matar a alguien y no querer matarlo: es posible que éste no sea nuestro fin último, pero no es posible que no se contemple como un objetivo (la intención indirecta es también intención). Ahora bien, que sea deductivo no quiere decir que sea necesariamente correcto, sino que el problema de su corrección se desplaza a la justificación de las premisas.

16. Para aumentar nuestra confianza en este argumento podemos insertar su conclusión en otro argumento similar: podemos aplicar el PRM no a la acción descrita como "disparar", sino descrita como "matar"25: El PRM nos dice ahora que si el agente mató intencionalmente, debía tener a su juicio alguna buena razón para matar. La conducta anterior y posterior del sujeto puede constituir indicios de esto. Por ejemplo, las discusiones anteriores entre Alfredo y Herminio pueden apuntar a que Alfredo tenía razones (motivos) para matar a Herminio.

También podemos servirnos en nuestras atribuciones de intenciones de cierta "lógica de la intención" derivada igualmente del PRM. Algunos de sus principios podrían ser los siguientes:

a) Si un agente tiene la intención de hacer A, y B es incompatible con A, no tiene la intención de hacer $\mathrm{B}$ (Principio de no contradicción de las intenciones). 
b) Si el agente cree que la acción B se sigue necesariamente de la acción A, no puede tener la intención de A y no tener la intención de B (Principio de transmisión de la intención a las consecuencias necesarias o previsibles).

c) Si el agente tiene la intención de hacer $B$ y cree que $A$ es el único medio para dar lugar a B, tiene también la intención de hacer A (Principio de transmisión de la intención a los requisitos causales o convencionales).

Por último, en el razonamiento con el que atribuimos intenciones necesitamos hacer referencia a las creencias del agente. Esto puede hacer pensar que hemos pasado del problema de atribuir intenciones al problema, igualmente difícil, de atribuir creencias. Sin embargo, esto es inevitable. Dado el rasgo que los filósofos llaman el "holismo de lo mental", es imposible definir un estado mental sin referencia a otro. En todo caso, quizá pueda pensarse que es más fácil atribuir creencias que intenciones, en el sentido de que son más obvias las máximas de experiencia que usamos. Ramón Ragués sugiere (entre otras) las siguientes:

a) La consideración de una persona como imputable lleva a atribuir a un sujeto todos aquellos conocimientos cuya ausencia sólo se entiende posible en quien padece algún tipo de perturbación psíquica o sensorial, o en menores de edad.

b) El hecho de que una persona haya sido normalmente socializada hace que se le puedan atribuir todos aquellos conocimientos cuya ausencia sólo se concibe en sujetos que no han tenido contacto con la civilización de la que se trate.

c) La circunstancia de que en un sujeto concurran determinadas características personales o de que ocupe una determinada posición social lleva a imputarle los conocimientos cuya ausencia haría impensable que tuviera esas características o que ocupara tal situación.

No obstante, a propósito de la atribución de creencias y de la evaluación de la situación hecha por el agente es preciso tener en cuenta los llamados errores del pensamiento cálido, como los estudiados por Elster y otros autores (la debilidad de la voluntad, el autoengaño, los posibles efectos aberrantes de la interacción entre deseos y creencias, como cuando atribuimos una exagerada probabilidad a aquello que deseamos que ocurra o la disminución de la fuerza del deseo en función de la dificultad, etc.) y la propensión a cometer ciertos errores en la atribución de probabilidades en contextos de incertidumbre o en ciertos tipos de razonamiento lógico. Es decir, el estudio de las perversiones de la racionalidad.

Se trata con ello de acercar la reconstrucción del razonamiento práctico que hizo el agente antes de actuar al que efectivamente realizó, y no al que hubiera realizado el "hombre medio" o el "hombre razonable". Estos dos últimos puntos de vista pueden ser relevantes para juzgar el grado de reproche (señalando que el sujeto pudo prever o debió prever ciertas consecuencias, por ejemplo), pero en una atribución descriptiva de intenciones sólo pueden tener una misión heurística y no deben actuar si tenemos razones para pensar que hubo un caso de evaluación equivocada de la situación.

17. ¿Cuál es la fundamentación del PRM y del resto de criterios de atribución de intenciones? No se trata de reglas de imputación de carácter normativo y ajenas a fines cognoscitivos, sino, al contrario, criterios de descubrimiento, algo así 
como "recetas" que tienden (con límites, por supuesto) a mostrar la corrección de nuestra atribución desde el punto de vista de una teoría de la verdad como correspondencia (entre la atribución y la realidad mental). El fundamento de estas "recetas" se basa en su éxito en el pasado (de hecho, como habrá observado el lector, no se trata de ningún criterio novedoso) como razonamiento cotidiano cada vez que atribuimos intenciones a los demás.

En el esquema de decisión que precede a muchas de nuestras acciones intencionales juega un papel importante el medio social en el que la acción va a realizarse. Lograr nuestros objetivos requiere en muchas ocasiones contar con la existencia de otros sujetos (entonces podemos hablar de nuestra acción como acción social). Nuestra evaluación de la situación debe tener en cuenta no sólo la existencia de tales sujetos, sino también su calidad de agentes que se mueven persiguiendo sus propios objetivos. Las acciones de los demás son importantes para nosotros si queremos coordinarnos con ellos, colaborar, competir o simplemente evitar sus interferencias. Para todo ello necesitamos atribuir intenciones a los demás, y es algo que hacemos cotidianamente.

Un gran número de estas atribuciones son exitosas, en el sentido de que logramos ajustar nuestra conducta a la de los demás y conseguir de esa manera nuestros objetivos. Si falláramos demasiado a menudo la vida en sociedad sería imposible y las más de las veces no alcanzaríamos nuestros objetivos (no sólo los objetivos colectivos, sino tampoco la mayoría de los individuales). Esta fiabilidad "en general" permite tener cierta confianza en nuestros criterios y procedimientos de atribución de intenciones y en nuestra manera de entender los conceptos involucrados (como el de intención), justificándolos desde un punto de vista práctico. Además, este tipo de justificación se basa en la experiencia: si en el pasado los criterios que uso para atribuir intenciones me han resultado generalmente provechosos no tengo razones para pensar que no vayan a seguir siéndolo en el futuro (aunque en casos concretos puedan conducirme a errores). Los criterios y el procedimiento de atribución no tienen por tanto un origen normativo en el mismo sentido en que lo pueden tener los criterios de atribución de responsabilidad moral o jurídica, por ejemplo, por lo que la concepción adscriptivista parece equivocada.

\section{BIBLIOGRAFÍA}

ACHInstein, Peter (1989), La naturaleza de la explicación, Fondo de Cultura Económica, México (trad. de Letizia García Urriza).

AlcÁcer Guirao, Rafael (2004), “Cómo cometer delitos con el silencio. Notas para un análisis del lenguaje de la responsabilidad", en VV.AA., Estudios penales en recuerdo del Profesor Ruiz Antón, Valencia, Tirant Lo Blanch.

ANDRÉs IBÁÑEZ, Perfecto (1992), Acerca de la motivación de los hechos en la sentencia penal, en Doxa. Cuadernos de Filosofía del Derecho, núm. 12, pp. 257-299. (http://www.cervantesvirtual.com/portal/DOXA/)

BAKER, Gerald y CLARK, Len (1994), La explicación. Una introducción a la filosofía de la ciencia, Fondo de Cultura Económica, México.

BAYÓn MohínO, Juan Carlos (1991), La normatividad del Derecho: Deber jurídico y razones para la acción, Centro de Estudios Constitucionales, Madrid. 
Bechtel, William (1991), Filosofía de la mente. Una panorámica para la ciencia cognitiva, Ed. Tecnos, Madrid (trad. de Luis Ml. Valdés Villanueva).

Belloch JulBe, Juan Alberto (1992), La prueba indiciaria, en Cuadernos de Derecho Judicial, XIII.

Bentham, J, (2001), Tratado de las pruebas judiciales, ed. Comares, Granada (trad. manuel Ossorio Florit).

BRonCANO, Fernando (1995), El control racional de la conducta, en Fernando Broncano (ed.), La mente humana, Enciclopedia Iberoamericana de Filosofía, ed. Trotta - Consejo Superior de Investigaciones Científicas, Madrid.

CAne, Peter (2000), Mens Rea in Tort Law, en Oxford Journal of Legal Studies, Vol. 20, núm. 4.

DAVIDSON, Donald (1995), Acciones, razones y causas, en Ensayos sobre acciones y sucesos, Ed. Crítica, Barcelona (trad. de Olbeth Hansberg, José Antonio Robles y Margarita Valdés).

Dennet, Daniel C. (1985), Mecanicismo y responsabilidad, Cuadernos de Crítica, México (trad. de Myriam Rudoy).

FEINBERG, Joel (1968), Action and responsibility, en Alan R. White, The Philosophy of Action, Oxford University Press.

Ferrajoli, Luigi (1997), Derecho y razón. Teoría del garantismo penal, Ed. Trotta, Barcelona (trad. de perfecto Andrés Ibáñez, Alfonso Ruiz Miguel, Juan Carlos Bayón Mohíno, Juan Terradillos Basoco y Rocío Cantarero Bandrés).

FleTCHER, George (1997), Conceptos básicos de Derecho penal, ed. Tirant lo Blanch, Valencia (trad. de Francisco Muñoz Conde).
García Amado, Juan Antonio (2000), ¿Dogmática penal sistémica? Sobre la influencia de Luhmann en la teoría penal, en Doxa. Cuadernos de Filosofía del Derecho, núm. 23, Alicante (http://www.cervantesvirtual.com/po rtal/DOXA/).

GARCía CARPINTERo, Manuel (1995), El funcionalismo, en Fernando Broncano (ed.), La mente humana, Enciclopedia Iberoamericana de Filosofía, ed. Trotta - Consejo Superior de Investigaciones Científicas, Madrid.

GARcía SuÁrez, Alfonso (1995), Qualia: propiedades fenomenológicas, en Fernando Broncano (ed.), La mente humana, Enciclopedia Iberoamericana de Filosofía, ed. Trotta - Consejo Superior de Investigaciones Científicas, Madrid.

GASCón, Marina (1999), Los hechos en el Derecho. Bases argumentales de la prueba, Marcial Pons, Madrid.

Giner, Salvador (1997), Intenciones humanas, estructuras sociales. Para una lógica situacional, en Manuel Cruz (ed.), Acción humana, Ed. Ariel, Barcelona.

Gómez Rodríguez, Amparo (1992), Sobre actores y tramoyas. La explicación situacional de la acción individual, ed. Anthropos, Barcelona.

- (1997), Microfundamentos de la explicación social, en Manuel Cruz (ed.), Acción humana, Ed. Ariel, Barcelona.

GonZÁlez LAGIER, Daniel (2001), Las paradojas de la acción (Acción humana, Filosofia y Derecho), Publicaciones Universidad de Alicante, Alicante.

- (2003-a), Hechos y argumentos. Racionalidad epistemológica y prueba de los hechos en el proceso penal (I), en Jueces para la democracia, núm. 46.

- (2003-b), Hechos y argumentos. Racionalidad epistemológica y prueba 
de los hechos en el proceso penal (II), en Jueces para la democracia, núm. 47.

HAmpshire, S. y Hart, H.L.A. (1958), Decision, Intention and Certainty, en Mind, vol. LXVII, núm. 265.

HART, H.L.A. (1948), The ascription of Responsability and Rights, en Proceedings of the Aristotelian Society, Londres.

HoRnsBy, Jennifer (1993), On What's Intentionally Done, en Stephen Shute, John Gardner y Jeremy Horder, Action and Value in Criminal Law, Clarendon Press, Oxford.

JAKOBS, Günther (1997), Sobre el tratamiento de los defectos volitivos y de los defectos cognitivos, en Estudios de Derecho penal, UAM Ediciones Civitas, Madrid (trad. de Enrique Peñaranda Ramos, Carlos J. Suárez González y Manuel Cancio Meliá).

JAKSON, Frank (2001), How Decision Theory Illiminates Assignments of Moral Responsibility, en Ngaire naffine, Rosemary Owens y John Williams, Intention in law and Philosophy, Dartmouth Publishing Company y Ashgate Publishing Company.

Laurenzo Copello, Patricia (1999), Dolo y conocimiento, Tirant lo Blanch, Valencia.

LIz, Manuel (1995), Causalidad y contenido mental, en Fernando Broncano (ed.), La mente humana, Enciclopedia Iberoamericana de Filosofía, ed. Trotta - Consejo Superior de Investigaciones Científicas, Madrid.

MASON, Anthony (2001), Intention in the Law Murder, en Ngaire Naffine, Rosemary Owens y John Williams, Intention in law and Philosophy, Dartmouth Publishing Company y Ashgate Publishing Company.

Miranda Estrampes, M. (1997), La mínima actividad probatoria en el proceso penal, J.M. Bosch Editor, Barcelona.

MosTERín, Jesús (1987), Racionalidad y acción humana, Alianza Editorial, Madrid.

Muguerza, Javier (1992) Presentación, en Amparo Gómez, Sobre actores y tramoyas. La explicación situacional de la acción individual, ed. Anthropos, Barcelona.

Paredes CAstañón, José Manuel (2001), Problemas metodológicos en la prueba del dolo, en Anuario de Filosofía del Derecho, XVIII.

Poggi, Francesca (2006) Proving Intention. Some General Remarks, en J. Ferrer Beltrán, M. Narváez Mora (eds.), Law, Politics and Morality: European Perspectives II. On Knowledge and Adjudication of National and European Law, Berlin, Duncker und Humblot, pp. 191-222.

RAGuÉs y VALLĖs, Ramón (1999), El dolo y su prueba en el proceso penal, J.M. Bosch Editor, Barcelona.

- (2002), Consideraciones sobre la prueba del dolo, En La Ley, Año XXIII, núm. 5633.

REDONDO, Cristina (1996), La noción de razón para la acción en el análisis jurídico, Centro de Estudios Constitucionales, Madrid.

RESCHER, Nicholas (1993), La racionalidad. Una indagación filosófica sobre la naturaleza y la justificación de la razón, Ed. Tecnos, Madrid (trad. de Susana Nuccetelli).

- (1994), Los límites de la ciencia, ed. Tecnos, Madrid (trad. de Leonardo Rodríguez Duplá).

- (1995), La lucha de los sistemas. Un ensayo sobre los fundamentos $e$ implicaciones de la diversidad filosófica, Universidad Nacional Autónoma de México, México (trad. de Adolfo García de la Sienra; revisión de Héctor Islas Azais). 
Rives Seva, Antonio Pablo (1999), La prueba en el Proceso Penal. Doctrina de la Sala Segunda del Tribunal Supremo, Ed. Aranzadi, Pamplona.

SEARLE, John (1985), Mentes, cerebros y ciencia, ed. Cátedra, Madrid (trad. de Luis Valdés).

- (1992), Intencionalidad, Ed. Tecnos, Madrid (trad. de Enrique Ujaldón Benítez; revisada por Luis Ml. Valdés Villanueva).

- (1996), El redescubrimiento de la mente, Ed. Crítica, Barcelona (trad. de Luis M. Valdés Villanueva).

- (2000-a), El misterio de la conciencia, Ed. Paidós, Barcelona (trad. de Antoni Domènech).

- (2000-b), Razones para actuar. Una teoría del libre albedrío, Ediciones Nobel, Oviedo (trad. de Luis M. Valdés Villanueva).

STOUTLAND, Frederick (1980), La teoría causal de la acción, en J. Hintikka, A. Macintyre, P. Winch y otros, Ensayos sobre explicación y comprensión, compilación de Juha Manninem y Raimo Toumela, Madrid, Alianza Editorial (trad. de Luis Vega).
TARUFFo, Michele (2002), La prueba de los hechos, Trotta, Madrid (trad. de Jordi Ferrer Beltrán).

- (2003), Algunas consideraciones sobre la relación entre prueba y verdad, en Daniel González Lagier y Jordi Ferrer Beltrán, Prueba, conocimiento y verdad, Revista Discusiones, Año III, núm. 3.

Toribio Mateas, Josefa (1995), Eliminativismo y el futuro de la psicología popular, en Fernando Broncano (ed.), La mente humana, Enciclopedia Iberoamericana de Filosofía, ed. Trotta - Consejo Superior de Investigaciones Científicas, Madrid.

VON WRIGHT, G.H. (1980-a), El determinismo y el estudio del hombre, en J. Hintikka, A. Macintyre, P. Winch y otros, Ensayos sobre explicación y comprensión, compilación de Juha Manninem y Raimo Toumela, Madrid, Alianza Editorial (trad. de Luis Vega).

- (1980-b), Freedom and Determination, Acta Philosophica Fennica, vol. XXXI, núm. 1.

- (1983), Explanation and understanding of Action, en Practical Reason, Philosophical Papers, vol. I, Basil Blackwell.

\footnotetext{
${ }^{1}$ Por todos, Ramón Ragués (1999), pp. 237 y ss. y Patricia Laurenzo Copello (1999), pp. 124 y ss. Como tal prueba indiciaria, debe someterse a determinados requisitos. Entre otros: (a) Los indicios a partir de los cuales se realiza la inferencia en nuestro caso, la conducta externa y sus circunstancias- deben obtenerse a partir de "prueba directa", esto es, no deben ser a su vez la conclusión de otra inferencia indiciaria (STS de 14 de Octubre de 1986; en contra de este requisito Belloch Julbe (1992), p. 67.); (b) los indicios deben conducir de modo unívoco a inferir el elemento interno, esto es, no debe existir una inferencia alternativa igualmente probable (Belloch Julbe (1992), pp. 70 y ss.).
}

2 STS de 20 de Julio de 1990.

3 Sobre el problema de los indicios-tipo en la prueba del dolo, véase Laurenzo Copello (1999), pp. 132 y ss.

4 Por ejemplo, STS de 2 de Febrero de 1988. Para una exposición y crítica de esta jurisprudencia véase Perfecto Andrés Ibáñez (1992), pp. 266 y ss.

5 Por todos, véase Ramón Ragués (1999) y (2002)

${ }^{6}$ No obstante, identificar las explicaciones con la indicación de causas (o factores que provocan) el fenómeno que se quiere explicar es sólo una aunque importante- de las concepciones posibles sobre la explicación. Para una introducción a otras 
concepciones de la explicación véase, por ejemplo, Gerald Baker y Len Clark (1994).

7 John Searle (2000-b), p. 125.

${ }^{8}$ Esta es una afirmación constante en la obra de von Wright. Véase, por ejemplo, von Wright (2002), p. 117.

${ }^{9}$ Esta es la tesis defendida por Donald Davidson (1995).

${ }^{10}$ La Tesis de la Conexión Lógica (no causal) entre intención y acción es defendida por von Wright (1987). Para una comparación de las dos Tesis puede verse Frederick Stoutland (1980).

${ }^{11}$ Esta misma idea se puede encontrar dentro de lo que Salvador Giner ha llamado la corriente sociológica principal, que asume los siguientes principios en cuanto a la explicación de la acción: "toda acción a) posee, para su autor, un significado subjetivo e intencional; b) no puede ser explicada racionalmente por quienes observan y analizan su desarrollo si tal significado no es tenido en cuenta; c) la explicación es posible, además, porque los seres humanos suelen actuar racionalmente, es decir, de acuerdo con su interpretación de la situación en que se encuentran (...)". Salvador Giner (1997). p. 40. Existe un cuarto presupuesto (de menor interés para nosotros): "Una acción es, además, social, si d) su intencionalidad tiene en cuenta la existencia de otro u otros seres humanos".

12 Para un estudio de los distintos modelos de explicación intencional de la conducta, con o sin Principio de Racionalidad, y para la propuesta de un modelo basado en un Principio de Racionalidad Mínima, véase Amparo Gómez Rodríguez (1992).

13 La adaptación es de Peter Achinstein (1989), p. 195.

14 Así lo interpreta Muguerza (1992), p. 8.

15 Amparo Gómez Rodríguez (1992), p. 27. Salvador Giner (1997), pp. 27 y ss.

16 Von Wright (1980-a), p. 185.

17 Von Wright ((1980-b), Freedom and Determination, p. 57.

${ }^{18}$ En realidad, los dos modelos son muy semejantes: la principal diferencia podría describirse como el hecho de que en von Wright el Principio de Racionalidad tiene carácter conceptual y no queda incluido en el argumento como una premisa, sino como la propia regla de inferencia.

${ }^{19}$ Que, en realidad, no son sino refinamientos de lo que podríamos llamar el "modelo de la psicología de sentido común", esto es, el que aplicamos cotidianamente al explicar y predecir la conducta de los demás recurriendo a sus creencias y deseos. Véase Josefa Toribio Mateas (1995), p. 246.

20 Von Wright, sin embargo, sí hace algunas consideraciones acerca de cómo verificamos las premisas del silogismo práctico (la intención y las creencias), para mostrar la conexión lógica entre éstas y la conclusión (la ocurrencia de la acción no puede verificarse si no es por referencia a la ocurrencia de las premisas y viceversa). Von Wright (1987), pp. 135 y ss.

${ }^{21}$ Así como la premisa que establece el Principio de Racionalidad debe ser aceptable empírica o conceptualmente o como un presupuesto epistemológico.

22 Nicholas Rescher (1995), p. 34.

23 Existe otro argumento general contra los intentos eliminacionistas y reduccionistas de identificar los estados mentales con procesos cerebrales o neuronales y dar prioridad a los segundos frente a los primeros. Este argumento ha sido expuesto por von Wright señalando que lo mental tiene prioridad epistémica frente a lo neuronal. Supongamos que deseamos establecer cuál es el estado neuronal que se corresponde con el estado mental "estar asustado" en un determinado sujeto. Si queremos investigar esto, debemos saber previamente que el sujeto se encuentra asustado, con algún criterio independiente de sus estados neuronales (es decir, observando su conducta externa). De manera que aunque lo neuronal tuviera prioridad causal frente a lo mental, lo mental tendría prioridad epistémica frente a lo neuronal (y la conducta externa tendría, según von Wright, prioridad semántica frente a lo mental). Von Wright (1996).

24 Taruffo (2003), p. 32.

25 Taruffo (2003), pp. 39 y ss.

${ }^{26}$ Laurenzo Copello (1999), p. 225. Este es el paso que parece dar también Ramón Ragués (aunque no sólo por razones ideológicas; también usando el argumento epistemológico), cuando afirma que "el recurso al sentido social implica que la consideración de una conducta como dolosa ya no depende de determinados datos psíquicos cuya aprehensión resulta imposible, tanto para el juez como para los ciudadanos, sino de que dicha conducta, de acuerdo con sus características externas y perceptibles, se valore socialmente como negación consciente de una concreta norma penal". Ramón Ragués (1999), p. 324. Un argumento similar es que la sentencia debe ser entendida, por lo que lo importante es adecuar sus resultados a la conciencia social. Esto nos lleva a que hay dolo cuando lo hay de acuerdo con la convicción social, y no cuando lo tiene realmente el sujeto.

27 Mosterín (1987), p. 193.

28 Por otra parte, cuando estas acciones tienen consecuencias lesivas o perjudiciales para terceros -cuando alguien roba por robar o mata por matartendemos a considerarlas patológicas en algún sentido y, por tanto, no intencionales o con una intencionalidad viciada.

${ }^{29}$ La noción de razón para la acción es enormemente complicada y no puedo entrar aquí a 
analizarla. Usaré la expresión en un sentido muy amplio y casi de "lenguaje común". Pueden encontrarse análisis en profundidad de esta noción en Juan Carlos Bayón (1991) y Cristina Redondo (1996).

30 John Watkins (1982), p. 127.

${ }^{31}$ Rescher (1993), p. 19.

32 Tomo esta denominación de Amparo Gómez Rodríguez, que define al Principio de Racionalidad Mínima como no actuar inconsistentemente. Gómez Rodríguez (1992), p. 129. En un sentido semejante, Salvador Giner escribe que "la acción humana es racional. Lo es en el sentido, y sólo en el sentido, de que persigue fines deseados por los sujetos según sus intenciones, recursos y creencias. Para ello los sujetos eligen, en todo tiempo y lugar, la senda disponible que juzgan más adecuada a ellos y a los recursos a su alcance". Salvador Giner (1997), p. 112.

${ }^{33}$ STS 1843/1999, de 23 de Diciembre.

${ }^{34}$ Entre acciones -o descripciones de accionesexiste una relación de generación: cuando disparo matando a alguien, la acción de disparar genera (causalmente) la acción de matar (otra manera de decirlo es afirmar que la relación de generación se da entre descripciones de acciones, y no entre acciones propiamente). Sobre esto véase Daniel González Lagier (2001).

${ }^{35}$ Ramón Ragués (1999), pp. 521 y ss.

${ }^{36}$ Fernando Broncano (1995), p. 311.

37 Salvador Giner (1997), p. 40.

38 De acuerdo con la hipótesis del animal maquiavélico, desde un punto de vista evolutivo la necesidad de reconocer los estados mentales de otros para adaptar la conducta propia y manipular la ajena en función de ellos fue fundamental para el desarrollo de la inteligencia y la racionalidad humana. Fernando Broncano (1995), pp. 320 y 321.

39 El criterio fiabilista o pragmático ha sido usado como criterio para justificar el conocimiento científico y la racionalidad epistemológica. Véase, por ejemplo, Rescher (1993), pp. 55 y ss. Para una aplicación del criterio al campo de la racionalidad práctica y evaluativa puede verse Fernando Broncano, (1995), pp. 326 y ss. 